\title{
Study on Evolution Model of Internet Public Opinion Based on Complex Network
}

\author{
Shibao Sun ${ }^{\mathrm{a}}$, Yanan Zhang ${ }^{\mathrm{b}}$, Jingshan Zhang ${ }^{\mathrm{c}}$, Chong Zhang ${ }^{\mathrm{d}}$ \\ College of Information Engineering, Henan University of Science \& Technology, Luoyang, China \\ asunshibao@haust.edu.cn, byn_haust@126.com, 'Superzhangjs@126.com, \\ dZhangchong1970@163.com
}

Keywords: Internet Public Opinion, Synergy Evolution, Order Parameter, Topology Structure

\begin{abstract}
In view of the complexity of Internet and the heterogeneity of individual in public opinion spreading, established the Internet public opinion evolution model based on complex network. Through experiment simulation, observed influences of order parameter and network topology on the evolution of opinion. Simulation results show that: 1 ) this model can greatly reflect the synergy effect of order parameters on opinion system, and it is more consistent with the actual situation. 2) the evolution of opinion is related to average degree and clustering coefficient: when average degree is small, the bigger clustering coefficient is, the more it can restrain the propagation of opinion; but when aveage degree exceeds a critical value, clustering coefficient no longer plays a key role, and at this point system will converge rapidly to extreme attitude.
\end{abstract}

\section{Introduction}

With the rapid development of information technology, Internet becomes the vital tool for people to obtain information, communicate with each other and express opinions, which brings great convenience to the production and propagation of public opinion. Fully recognizing the evolution law and the transmission mechanism of public opinion can help social managers grasp social conditions and public opinions. It has very important realistic meanings to control the public opinion guidance and defuse the crisis of public opinion.

At present, the study of opinion evolution can be classified into two types. One is the bottom-up microcosmic particle opinion dynamics models, mainly include Sznajd-Weron model [1]、Deffuant model [2], etc.. The other is the top-down macro modeling method. This class of models include traditional epidemic model [3], chaos and synchronized emergence model [4] and synergistic opinion model [5-8], etc. Based on modern mathematical theories, synergy is a systematic theory that can quantitatively describe the evolution rule of the system from disorder state to order state. Through establishing social public opinion model, Ref. [5] elaborated the synergy phenomenon of the opinion from disorder state to order state. Ref. [6] applied synergetic rule to local five neighbors of cellular automata. Ref. [7, 8] extended five neighbors to nine neighbors, considered global and local synergetic transition probability and studied the effect of order parameter on opinion transmission.

As the media of opinion information dissemination, Internet is a typical complex social network, which plays an important role in opinion dissemination and evolution. In Ref. [7, 8], individuals are considered homogeneous, cellular neighborhood are limited in the fixed area. And the treatment of interpersonal relationships in opinion dissemination is too simple. This paper introduces complex network theory into the synergetic opinion evolution, expands the neighborhood structure of individual interaction, establish synergetic opinion evolution model on complex network.

\section{The construction of opinion evolution network}

This paper adopts network model [9] Holme\&Kim proposed. By adding a triad formation step in the growth process of the BA scale-free network, the scale-free network with tunable clustering is constructed as carrier of the opinion evolution. The construction algorithm is as follows: 


\section{Algorithm.1:}

1) Growth: To start with, the network consists of $m_{0}$ vertices. One vertex $i$ with $m$ edges is added at every time step $\left(m \leq m_{0}\right)$;

2) Preferential attachment: Each edge of $i$ is then attached to an existing vertex $j$ with the probability proportional to its degree, i.e. the probability for a vertex $j$ to be attached to $i$ is

$p=\frac{k_{j}}{\sum k_{v}}\left(k_{j}\right.$ is the degree of vetex $\left.j\right)$;

3) Triad formation: If an edge between $i$ and $j$ was added in the previous step, then add one more edge from $i$ to a randomly chosen neighbor $r$ of $j$ with the probability $p t$. If all neighbors of $j$ were already connected to $i$, do a preferential attachment step instead until $m$ edges is added.

\section{The Model}

We pay attention to the synergistic effect between network topology and node dynamics behavior, and construct the synergistic opinion evolution model on complex networks. $G=(V, E)$ generated by Algorithm.1 represents the network of opinion evalution, where $V$ is the node set, $E$ is the edge set. Nodes on behalf of Internet users, the total number recorded as $N=|V|$, while edges represent the adjacent relationship between individuals. Further, the neighbor relation of the nodes can be given by the network adjacency matrix $A$. In matrix A, column vector $i$ represents the neighborhood of node $i$, denoted as $L_{i}=\left\{a_{i j} \mid a_{i j} \in A, j=1,2, \ldots, N\right\}$. Where $a_{i j}=1$ denotes there is a link between node $i$ and $j$; where $a_{i j}=0$ denotes there isn't a link between node $i$ and $j$. The discrete state set $S=\{+1,-1\}$ is defined as opinion set, where $s_{i}(t)=+1$ denotes node $i$ in favor of, where $s_{i}(t)=-1$ denotes node $i$ is of opposition at time step $t$.

According to Hacken synergy principle [5], the probability of attitude transformation at next time step can be given by the follow equations :

$$
\begin{aligned}
& P_{(+1 \rightarrow-1)}=v \cdot \frac{e^{-(k q+h)}}{e^{k / 2}} \\
& P_{(-1 \rightarrow+1)}=v \cdot \frac{e^{k q+h}}{e^{k / 2}}
\end{aligned}
$$

The two equations are considered as the synergy transition probability of individual attitude, where Eq. 1 represents the probability of individual attitude transformation from pro attitude $(+1)$ to opposition attitude(-1); where Eq.2 represents from opposition attitude(-1) to pro attitude $(+1)$. Parameter $v, k$ and $h$ are used as the order parameter in the process of public opinion synergy evolution, which reflect the strength of synergy effect. Where $v$ is defined as self changing rate, parameter $v$ take value of 0 generally. Where $k$ is defined as the order-variable parameters of society adaptability, represents individual's compliance level to majority opinion. If $k$ is bigger, individual is more greatly influenced by public opinion environment, and $k \in[0,10]$. Where $h$ is defined as the preference to pro attitude $(+1)$ or opposition attitude(-1), and $h \in[-1,+1]$.

Different from the Refs. $[6,7,8]$, the opinion imbalance degree in this paper is defined as $q$ :

$$
q=\frac{n_{+}-n_{-}}{2|L|}, n_{+}+n_{-}=|L|
$$

$|L|$ is the amount of the current node's neighborhood set, that is the node's degree in the network of (each node's degree is not the same). $n_{+}$is the number of netizen who holds pro attitude $(+1)$ in the neighborhood, $n_{-}$is the number of netizen who holds opposition attitude(-1) in the neighborhood, and $q \in[-0.5,+0.5]$.

To measure the extent of the whole public opinion system tend to favor or oppose, the magnetisability $M(t)$ of system at time $t$ is defined as: 


$$
M(t)=2 p_{+}(t)-1
$$

Where $p_{+}(\mathrm{t})$ represents the proportion of persons with pro attitude $(+1)$ in opinion network at time $t$.

\section{Simulation and Analysis}

Using simulation tools Matlab R2012b,take the above-mentioned model to update asynchronously by Monte Carlo simulation. The simulation experiment is divided into two parts: First, verify the rationality and effectiveness of the model; Second, the study mainly focuses on the influence of network topology characteristics on synergistic effect, and do in-depth analysis combined with previous step of work.

Specific simulation process is as follows:

Step1: Initialize parameter set $N 、 m_{0} 、 m 、 p$ and $p_{+}, v, k, h$, which $v$ to take a fixed value of 0.5. The network topology structure $G$ and its adjacency matrix $A$ of public opinion evolution are generated by the Algorithm.1 , and the initial state of $N$ individuals are assigned randomly according to the proportion of $p_{+}:\left(1-p_{+}\right)$.Simulation step $T=100$, initial time $t=0$;

Step2: At each time step, randomly choose a node $i$ from $G$, and calculate neighborhood set $L_{i}$ of node i from $A$, then calculate imbalance degree $q$ according to the Eq.3. At this point, if $s_{i}(t)=+1$, by the Eq. 1 to calculate the synergistic transition probability $P_{(+1 \rightarrow-1)}$, and to change the attitude with probability $P_{(+1 \rightarrow-1)}$; if $s_{i}(t)=-1$, change one's attitude with probability calculated by Eq.2. Until all nodes in $G$ are selected, such one traversal is referred to as a simulation time step $t$;

Step3: Calculate the proportion $p_{+}(t)$ of individual who hold pro attitude at time $t$, and calculate and record system magnetic susceptibility $M(t)$ at time $t$ according Eq.4;

Step4: Repeat Step2、3, until it reaches a preset maximum time step T;

Step5: Output system magnetic susceptibility $M$ versus time curve, the simulation ends.

Effect of order parameter on opinion evolution. First take $N=2500 、 m_{0}=7 、 m=4 、 p_{t}=0.5$, construct the network structure of public opinion evolution. Order $p_{+}=0.35$, at this time the initial magnetisability is $M_{(t=0)}=-0.3$, that is, opposition attitude is dominant opinion. when $h=0$, set the parameter $k \in\{0,1,2,3,4,8\}$, when $k=2$, set the parameter $h \in\{0,0.2,0.4,0.6,0.8,1.0\}$. Through simulation experiments, investigate magnetisability under different $k$ and $h$, the results are as shown in Fig.1 and Fig.2.

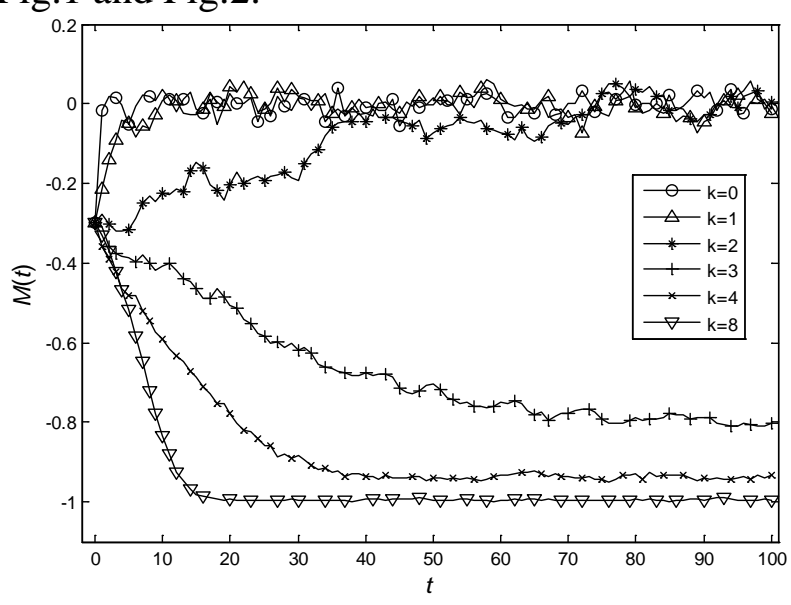

Fig.1 Magnetisability curve for $h=0$ and different $k$

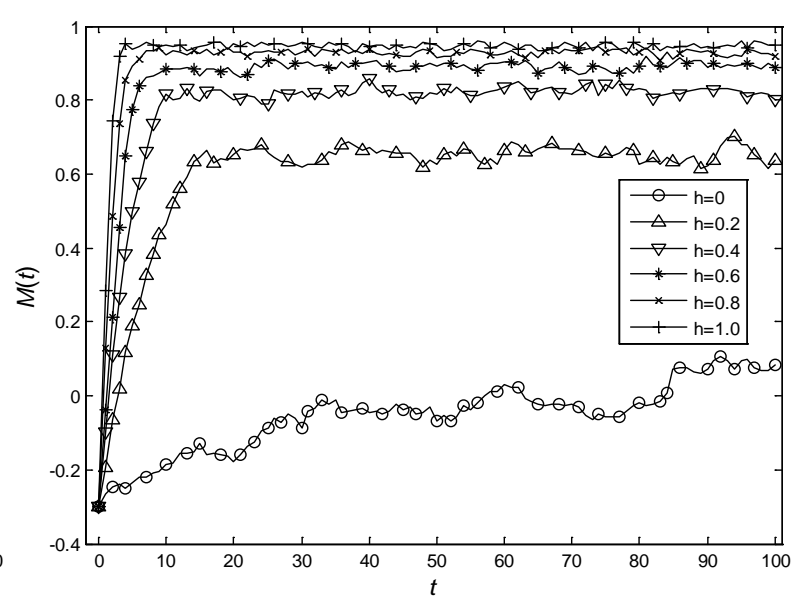

Fig.2 Magnetisability curve for $k=2$ and different $h$

Fig. 1 depicts the system magnetisability curve for $h=0$ and different $k$ value. When $k \leqslant 2$, whole system society adaptability level is relatively lower, individual attitude had a trend to change freely at this time. So when the final state of the system, the magnetisability curves always shocks near the zero line. When $k>2$, the system exhibits obvious phase transition, magnetisability quicly close to the mainstream attitude. After the 40 time steps, magnetisabilit basically stabilize at between -0.8 and -1 , the overall system shows a strong opposition state. Fig. 2 depicts the magnetisability curve for $k=2$ 
and different $h$ value. When $h=0$, individual attitude not to favor any party, magnetisability showing a state of uncertainty; when $h>0$, magnetisability changes towards positive direction, and $h$ is the greater, magnetisability level is higher. When $h=0.9$, the magnetisability is properly close to +1 , the system trend pro attitude. The above analysis shows that the model can not only reflects order parameter's reinforcement effects to system, but also shows some features that traditional synergistic opinion model does not have.

Effect of network topology on magnetisability. Opinion evolution is affected by network structure, this section focuses on the role of the average degree $<k>$ and clustering coefficient $C$ in opinion synergistic evolution. Here make $k, h$ takes the value of the phase transition point respectively, that is $k=2 、 h=0$. Meanwhile, set $N=1000 、 m_{0}=7 、 m \in\{2,3,4,5,6,7\}, p_{t} \in\{0.1$, $0.3,0.5,0.7,0.9\}, p_{+}$still take 0.35 . First, let $m$ take the value of $\{2,3,4,5,6,7\}$, and then generate six different networks which have different average degree $<k>$ according to an Algorithm.1. In scale-free networks with tunable clustering, $\langle k\rangle \approx 2 m$, so that $\langle k\rangle$ of those networks are approximately 4, 6, 8, 10,12, 14. Thereafter, adjust $C$ of above-mentioned networks through different $p_{t}$, and the corresponding relation between $<k>$ and $C$ is shown in Fig.3. Fig.3 shows that, in those networks which $<k>$ is different from each other, the $C$ and $p_{t}$ present a positive correlation.

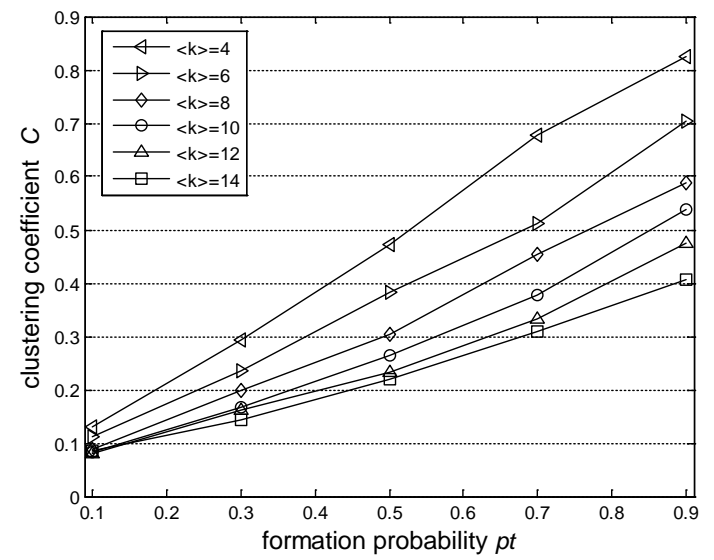

Fig.3 Networks clustering coefficients under different triad formation probability $p_{\mathrm{t}}$

Then make experiments with those networks, the results are shown in Fig.4. As can be seen from Fig.4(a),(b),(c), when $<k>\leqslant 8$, with increasing $C$, but magnetisability decreases gradually, indicating that the higher $C$ plays an key role on the polarization of opinion. Fig.4 (d), (e), (f) shows that when $<k>>8$, system will quickly tend to stable state. At this time, no matter how much $C$, magnetisability can achieve -1 . This shows that when $<k>$ exceeds the critical value of 8 , the system will accelerate the convergence of the opinion polarization state, and then $C$ no longer plays a key role in evolution.

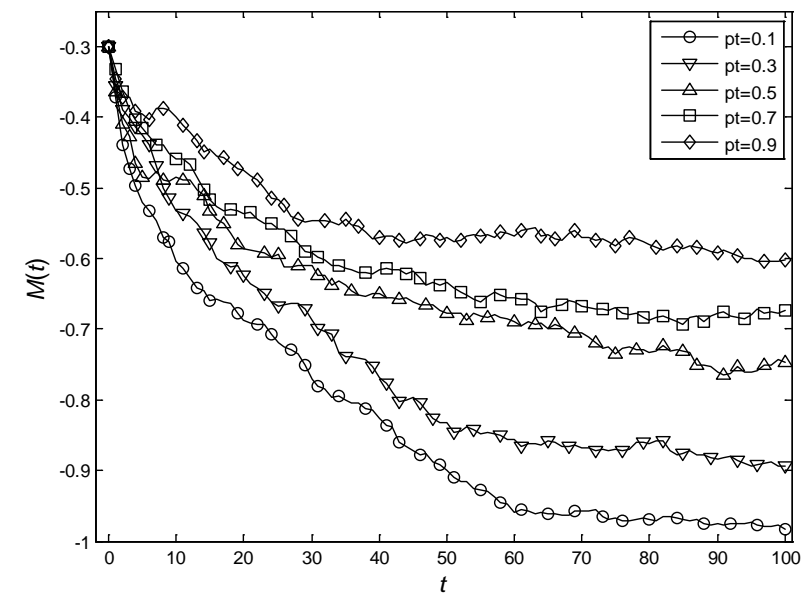

(a) $\langle k\rangle=4$

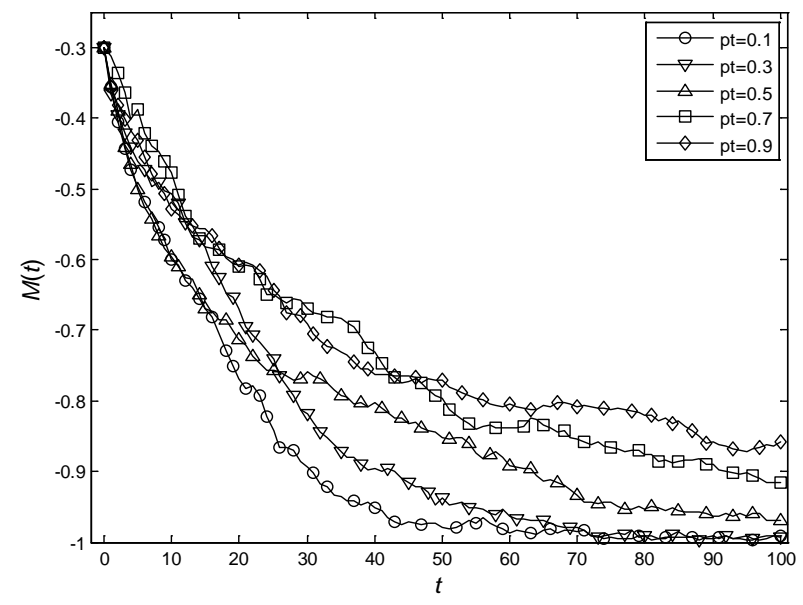

(b) $\langle k\rangle=6$ 


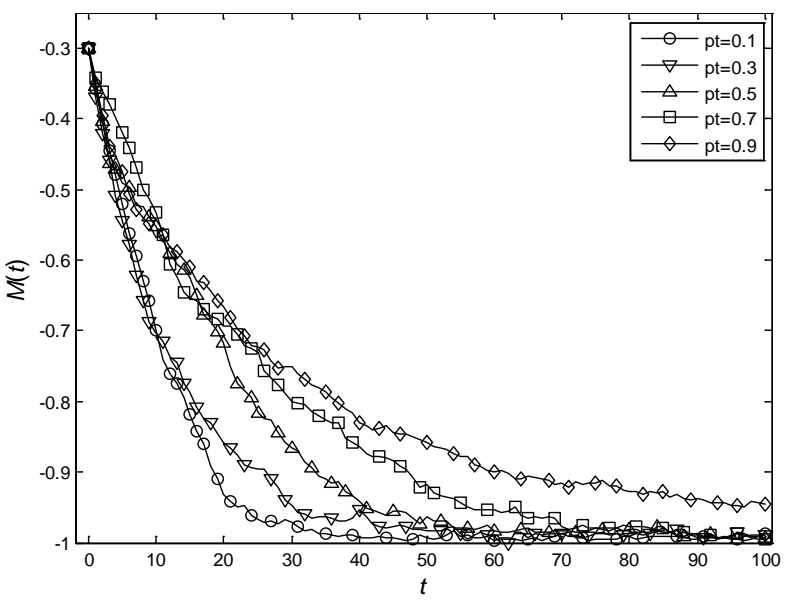

(c) $\langle k\rangle=8$

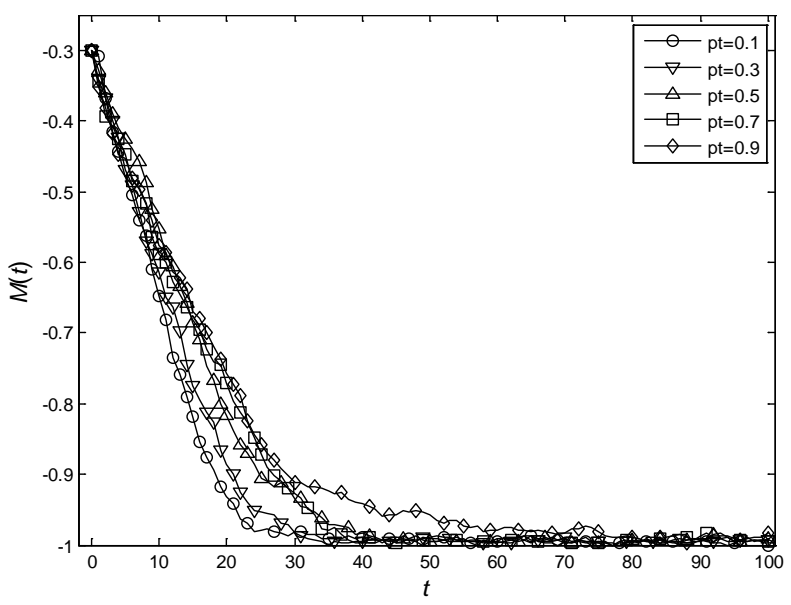

(e) $\langle k\rangle=12$

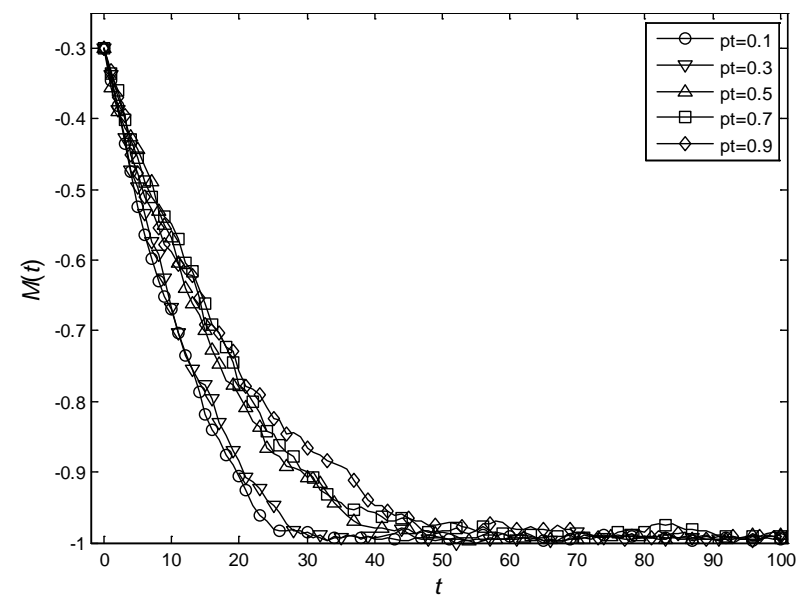

(d) $\langle k\rangle=10$

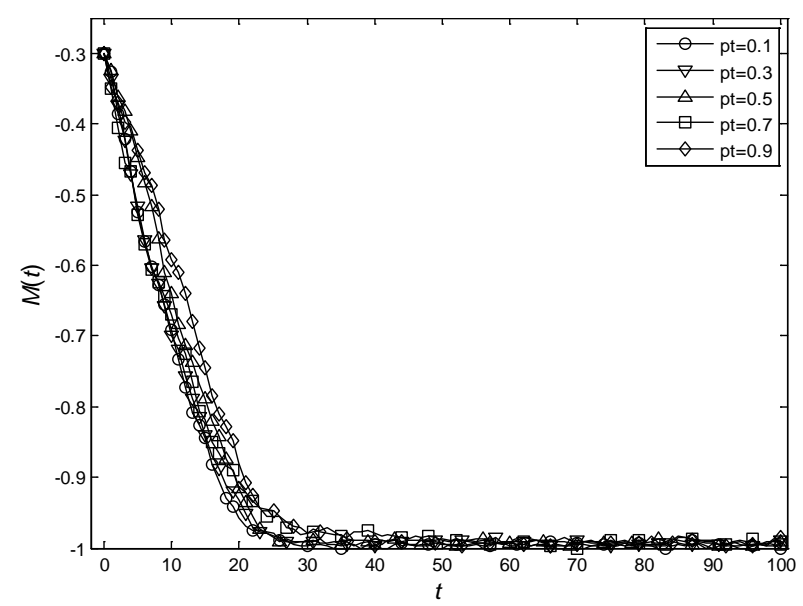

(f) $\langle k\rangle=14$

Fig.4 Effect of clustering coefficient on magnetisability under different network averages

The above discussion is basically in accord with the reality: The higher clustering coefficient means that the synergy among netizens is more higher, it is beneficial to strengthen sincere communication and coordination between netizens, which can enhance the transparency of public opinion information to some extent, thus the false information is limited to a certain range. However, when the number of netizens' friends is so large that break a threshold value, the whole network will become more intensive, and the frequency of interaction between individuals is higher as well. The number of people who concern about public events is bound to increase, which will exacerbate the spread of rumors and false information.

\section{Conclusion}

This paper overall considered the Internet environment of opinion transmission and the heterogeneity of individual interactions, established the synergetic opinion evolution model. Through theoretical analysis and simulation experiments, the following conclusions are drawn:

1) Order parameter $h, k$ have a significant impact on the synergy effect of public opinion. In the process of evolution, there is a phase transition point of the order-variable parameters of society adaptability $k$, preference $h$ is still the key factor to determine the trend of public opinion. In addition, under the influence of the heterogeneity degree distribution, the oscillation of system magnetic susceptibility is more obvious, which reflects the synergy effect of public opinion system to a greater extent.

2) The network structure characteristics have moderation effects on magnetic susceptibility of the system. When the network average degree $<k>$ is less than the critical value, the network connection is relatively sparse. At this time, improving the clustering coefficient is conducive to the suppression 
of the spread of public opinion. When the average degree $<k>$ is more than a critical value, the network connection is becoming more closely. At this time, increase the average degree $<k>$ will accelerate the magnetisability to reach the maximum, which lead to the whole system tends to some extreme attitude, and the influence of clustering coefficient almost disappeared.

\section{Acknowledgement}

In this paper, the research was sponsored by Supported byProgram for Innovative Research Team (in Science and Technology) in University of Henan Province (Project No.17IRTSTHN010), Technological Innovation Team of Henan University of Science and Technology (Project No.2015XTD011) and Major Cooperative Engagement Fund of Henan University of Science and Technology (Project No.2015ZDCXY03), Research on science and technology project of Henan Province (Project No. 152102210277), Project of industry university research cooperation in Henan Province (Project No. 152107000027).

\section{References}

[1] Sznajd-Weron K, Sznajd J. Opinion evolution in closed community. International Journal of Modern Physics C, Vol. 11(2000): p.1157- p.1165.

[2] Guillaume D, David N, Frederic A. Mixing beliefs among interacting agents. Advances in Complex, Vol. 3(2000): p.87- p.98.

[3] Zhu G H, Jiang G P, Xia L L. Rumor Spreading Model Considering Conformity Phenomena in Complex Social Networks. Computer Science, Vol. 43(2016): p.135- p.143.

[4] Zhang Y F, Xiao R B. Emergence mechanism of consensus synchronization in internet collective behavior based on cellular automata. System Engineering Theory and Practice, Vol. 34(2014): p. 2600- p. 2608.

[5] H.Hacken. Synergetics. Xu Xishen,Chen Shigang,Chen Yashen,etc. BeiJing:Atomic Energy Press. 1984: p. 399- p. 402.

[6] Zeng X K. Study of the Cellular Automaton Models of Public Opinion Based on Majority Rule and Cooperation Rule. GuiLin: Guangxi Normal University, 2007.

[7] Fang W, He L J, Song L T. Synergistic cellular automata model for dissemination of Internet public opinion. Journal of Computer Applications, Vol. 32(2012):p. 399-p. 402.

[8] Fang W, He L j, Song L T. Predictive Modeling \& Simulation for Propagation of Internet Public Opinion. Computer Science, Vol. 39(2012): p. 203- p. 235.

[9] Holme P,Kim B J. Growing scale-free networks with tunable clustering. Physical Review E, Vol. 65(2002):026107. 